\title{
Bloodstream Infection and Endocarditis Caused by Staphylococcus aureus in Patients with Cancer: A Multicenter Cohort Study
}

\author{
Sara Grillo · Guillermo Cuervo · Júlia Laporte-Amargós • \\ Manel Tuells · Immaculada Grau • Dàmaris Berbel • Carlota Gudiol • \\ Miquel Pujol · Jordi Carratalà
}

Received: October 8, 2021 / Accepted: November 24, 2021 / Published online: December 2, 2021

(C) The Author(s) 2021

\section{ABSTRACT}

Introduction: In a large cohort of patients with Staphylococcus aureus bloodstream infection (SABSI), we aimed to analyze the incidence and risk factors for infective endocarditis (IE) among

Supplementary Information The online version contains supplementary material available at https:// doi.org/10.1007/s40121-021-00575-8.

S. Grillo · G. Cuervo $(\bowtie) \cdot J$. Laporte-Amargós · M. Tuells · I. Grau · C. Gudiol · M. Pujol ·

J. Carratalà

Department of Infectious Diseases, Bellvitge University Hospital, IDIBELL, Feixa Llarga S/N, 08907 L'Hospitalet de Llobregat, Barcelona, Spain e-mail: guillermo.cuervo@bellvitgehospital.cat

J. Laporte-Amargós · C. Gudiol

Infectious Diseases Unit, Hospital Duran y Reynals, Institut Català d'Oncologia (ICO), Barcelona, Spain

D. Berbel

Department of Microbiology, Bellvitge University

Hospital, IDIBELL, Barcelona, Spain

C. Gudiol · J. Carratalà

University of Barcelona, Barcelona, Spain

I. Grau $\cdot$ D. Berbel

Centro de Investigación Biomédica en Red (CIBER) de Enfermedades Respiratorias, Instituto de Salud Carlos III, Madrid, Spain

C. Gudiol · M. Pujol · J. Carratalà

Centro de Investigación Biomédica en Red (CIBER) de Enfermedades Infecciosas, Instituto de Salud Carlos III, Madrid, Spain patients with active cancer (PAC) in comparison with those without cancer (PWC).

Methods: Multicenter cohort study of patients with SABSI admitted to two tertiary care hospitals, from 2011 to 2019. PAC were defined as those with an active solid organ cancer or hematological malignancies. SABSI and S.aureus IE were compared between PAC and PWC. Results: Among 978 episodes of SABSI, 217 (22.2\%) occurred in PAC. PAC were younger, had fewer comorbidities, carried cardiac devices less often, and had less community-acquired SABSI than PWC. Compared to PWC, PAC more frequently had catheter-related SABSI, less IE $(2.8 \%$ vs $10.9 \%, p<0.001)$ and osteoarticular infection $(2.3 \%$ vs $14.3 \%, p<0.001)$. Independent risk factors for IE were cardiopathy (aOR $4.392,95 \%$ CI 2.719-7.094) and persistent bacteremia (aOR 3.545, 95\% CI 2.159-5.820). Thirty-day mortality was high, and similar between groups $(24.2 \%$ vs $25.5 \%, p=0.282)$.

Conclusions: PAC with SABSI developed IE less frequently than PWC did. This finding seems related to the differences in baseline characteristics and may have significant clinical implications, such as transesophageal echocardiography in PAC without cardiopathy or persistent bacteremia.

Keywords: Staphylococcus aureus; Endocarditis; Bloodstream infection; Cancer 


\section{Key Summary Points}

The incidence of $S$. aureus endocarditis was lower in patients with cancer compared to patients without cancer.

Risk factors for endocarditis were previous cardiopathy and persistent bacteremia.

Key baseline differences explain lower incidence of endocarditis in patients with cancer.

Transesophageal echocardiography could be avoided in patients with cancer without cardiopathy or persistent bacteremia.

\section{INTRODUCTION}

Staphylococcus aureus is one of the leading causes of bloodstream infection (BSI) worldwide [1]. Healthcare exposure and invasive procedures increase the burden of this disease [2]. Furthermore, S.aureus is ranked among the most important causes of bloodstream infection-associated death [3]. Mortality of patients with $S$. aureus bloodstream infection (SABSI) has been consistently associated with host and pathogen characteristics as well as with prompt source management and adequate antibiotic treatment [3]. One of the most important aspects among the host-pathogen interaction is the source of the infection. Among these, endocarditis and pneumonia are the ones with the highest associated mortality rates [3]. S. aureus causes around $25 \%$ of episodes of infective endocarditis (IE) [4]. In a recent Danish nationwide study including 69,000 bacteraemia episodes, IE was diagnosed in one of every 10 SABSI episodes [5], and it has also been identified as a strong risk factor for 30-day mortality in patients with SABSI $[6,7]$.

Cancer incidence and mortality are rapidly rising worldwide [8]. Bloodstream infection (BSI) entails an important risk of complications in patients with malignancy. Indeed, cancer is the most frequent comorbidity in patients with sepsis, described in $17 \%$ of cases [9]. BSI in patients with cancer may delay the start of chemotherapy, increase the length of hospitalization and costs, and raise morbidity and mortality rates [10]. SABSI is a serious complication in patients with cancer, and is a clear risk factor for mortality, with an odds ratio (OR) of 1.8 (95\% CI 1.03-3.15) [11].

To date, few studies have specifically focused on SABSI in patients with cancer, even though it is the second leading cause of Gram-positive BSI [12]. SABSI has usually been associated with gastrointestinal mucositis and catheter-related infection [13, 14]. Nosocomial acquisition, urinary catheter, nasogastric tube, corticosteroids, skin and soft tissue infection, and pneumonia are also significantly associated with SABSI in patients with malignancy [11]. The incidence of IE in patients with cancer and SABSI seems to be lower than in patients without cancer, but this issue has not been explored in depth in the literature $[15,16]$. To the best of our knowledge, no studies have focused on IE in this specific population. Nor have the characteristics of SABSI in patients with cancer been compared with those of SABSI in other patients, and it is not clear at present why patients with cancer appear to be less prone to IE than patients without malignancies.

In our large multicenter cohort of SABSI, we aimed to analyze the epidemiology, clinical characteristics, risk factors for IE, and outcomes of patients with active cancer (PAC) in comparison with those without malignancies (PWC).

\section{METHODS}

\section{Study Design, Setting, and Participants}

The study design consisted in a retrospective cohort analyses of prospectively collected data of SABSI episodes in adult patients (at least 18 years old) hospitalized from January 2011 to December 2019 in two tertiary care hospitals in Barcelona, Spain: Bellvitge University Hospital, a 700-bed teaching hospital for adults, and 
Duran i Reynals Hospital, a 200-bed referral cancer center. Positive blood cultures were reported daily by members of the Microbiology Department. All patients were prospectively followed up by infectious disease specialists during hospital admission and for up to 90 days thereafter.

This study was approved by the Clinical Research Ethics Committee of Bellvitge University Hospital (PR326/21). The informed consent form and information sheet were waived because of the retrospective nature of the study. Patient data were anonymized for the purposes of the analysis. Information that could identify patients was protected according to the national normative approach.

Some parts of the methodologies and analyses used in this study were similar to those published in some of our previous studies, reported elsewhere $[17,18]$.

\section{Variables and Data Sources}

Demographic, epidemiological, clinical, and microbiological data were prospectively collected from patients with SABSI. For each patient, the following data were recorded: age, sex, comorbidities, functional and immunological status, history of contact with healthcare providers or prior antibiotic therapy, implanted foreign bodies or devices, clinical data at the BSI onset (grade of severity of illness at presentation, source of infection, and the presence of septic emboli), diagnostic and therapeutic interventions, antibacterial therapy, and outcomes. Follow-up information for up to 90 days after BSI was obtained by reviewing the patients' electronic clinical charts, in order to assess the mortality and microbiological failure rates.

\section{Bias}

All consecutive episodes of SABSI detected during the study period were included in the initial analysis with the objective of minimizing selection bias.

\section{Definitions}

SABSI was defined as at least one positive blood culture obtained from a patient with clinical signs and symptoms of infection (namely fever, chills, malaise, specific signs or symptoms of infection, e.g., phlebitis, cellulitis, septic emboli). The presence of underlying diseases was assessed by the Charlson comorbidity index [19]. BSI acquisition categories were considered in three mutually exclusive classes, communityacquired, nosocomial, and healthcare-related, based on the criteria from Friedman et al. [20]. Sepsis was assessed with the quick Sequential Organ Failure Assessment (qSOFA) score, and was defined as a score of at least 2 points [21].

The source of infection was defined as the infective focus possibly responsible for the BSI, based on the clinical presentation, physical examination, and complementary results. The sources of infection included eight groups: vascular catheter-related infections; infective endocarditis (according to the modified Duke criteria) [22]; skin and soft tissue infections; pneumonia; osteoarticular infections (with or without prosthetic devices); urinary tract infections; unknown; and other sources (including abdominal, head and neck, and central nervous system sources). IE was considered for both source of infection and complication of BSI from another source.

Platelet count was assessed at the time of hospitalization and confirmed with a second blood count performed 48-72 h after hospital admission. Previous or concomitant use (for at least 3 months before SABSI) of antiplatelet and anticoagulant drugs was recorded. Echocardiography was performed in all patients except those who presented rapid clearance of bacteremia.

Persistent bacteremia was considered when follow-up blood cultures after more than 2 days of treatment were positive. Cases of positive blood culture after negative follow-up blood cultures were considered recurrent bacteremia. We considered consecutive episodes where bacteremia occurred after the 90-day follow-up 
and after clear resolution of the previous episode. Source control refers to all the physical procedures used to control a focus of infection, including drainage of liquid/purulent abscesses, debridement of necrotic tissues, removal of indwelling catheters and potentially infected devices, and we considered this achieved if performed within the first $72 \mathrm{~h}$ from BSI.

Cardiopathy was defined as the presence of one or more of the following conditions: previous predisposing factor for IE heart conditions such as valvular heart disease and/or presence of cardiac devices. Valvulopathy or valvular heart disease was defined as the presence of previous damage in any cardiac valve. Patients with active cancer (PAC) were defined as those with active solid organ cancer or hematological malignancies, who underwent chemotherapy/ radiotherapy and/or hematopoietic stem cell transplant in the previous 3 months or who had metastatic cancer. SABSI and $S$. aureus IE in PAC were compared with patients without cancer (PWC). Mortality was defined as all-cause death and was evaluated 30 days after bacteremia onset.

\section{Microbiological Studies}

Blood cultures were processed in a BD BACTEC $^{\mathrm{TM}}$ FX blood culture system (Becton Dickinson, Barcelona, Spain) at the Department of Microbiology of Bellvitge University Hospital. In the first 2 years of the study (2011-2012), $S$. aureus was identified by latex agglutination tests (Pastorex Staph-plus, Bio-Rad Laboratories, Madrid, Spain) and DNase production (DNase test agar, bioMérieux, Marcy l'Etoile, France). From 2013 onwards, identification was carried out by MALDI-TOF (MALDI Biotyper; Bruker Daltonics, Spain). Antimicrobial susceptibility was determined by microdilution using commercially available panels (MicroScan, Beckman Coulter, Barcelona, Spain) and according to the EUCAST guidelines [23]. To rapidly assess methicillin resistance, following positivity of the blood culture and the confirmation of S. aureus isolation, Xpert ${ }^{\circledR}$ MRSA/SA Blood Culture (Cepheid, Sunnyvale, CA) was used.

\section{Statistical Methods}

To calculate the incidence of SABSI and IE, we divided the number of episodes of SABSI and IE, respectively, by the annual hospital discharges. Categorical variables are presented as numbers of episodes and percentages, and continuous variables as means and standard deviation or medians and interquartile range (IQR). Continuous variables were compared using the Student's $t$ test or Mann-Whitney $U$ test as appropriate. Fisher exact test or Pearson $\chi^{2}$ test was applied to assess the relationship between categorical variables. All consecutive episodes of SABSI detected during the study period were included in the analysis in order to minimize selection bias.

Multivariate logistic regression analysis was then used to assess factors potentially associated with IE and with 30-day mortality, and included all significant variables identified in the univariate analysis along with the relevant ones according to the literature. The goodness of fit of the model was assessed by the Hosmer-Lemeshow test. Relative risks are expressed as adjusted odds ratios (aORs) and 95\% confidence intervals. Statistical significance was established at $\alpha=0.05$. All reported $p$ values are two tailed.

\section{RESULTS}

\section{Participants and Descriptive Data}

During the study period, 978 episodes of SABSI were recorded and followed up. Of these, 761 (77.8\%) occurred in PWC and 217 (22.2\%) in PAC. Among PAC, 104 (47.9\%) had solid organ cancer and 113 (52.1\%) had hematological malignancies. Among solid organ cancer, patients had more frequently head and neck (13.4\%), breast $(9.6 \%)$, and colorectal cancer $(8.6 \%)$. The hematological malignancies most frequently identified were myeloid acute leukemia (23\%), multiple myeloma (14\%), diffuse large B cell lymphoma (8\%), and T cell lymphomas (8\%). Seventeen patients (15\%) had previously undergone hematopoietic stem cell transplantation. Seventy PAC (32.2\%) presented 
neutropenia (neutrophil level less than $500 / \mu \mathrm{L}$ ) at SABSI presentation, while in five $(2.3 \%)$ citomegalovirus infection was documented and seven patients presented invasive fungal infection $(3.2 \%)$. The incidences of SABSI are shown in Fig. 1: the incidence of SABSI was higher in PAC than in PWC (7.3/1000 hospital discharges/year vs. 2.9/1000 hospital discharges/ year, $p<0.001$ ).

Baseline characteristics and differences between PAC and PWC are shown in Table 1. Overall, there were 647 men (66.3\%), mean age was 64.6 years, and the patients had a mean Charlson comorbidity index of 5.4 points. PAC were younger, had fewer chronic comorbidities, and they were less likely to receive antiplatelet drugs. Acquisition of SABSI was different between PAC and PWC $(p<0.001)$ : community-acquired SABSI was more common in PWC than in PAC (7.4\% vs $28.0 \%)$, while PAC had more healthcare-related (54.4\% vs $41.7 \%)$ and nosocomial SABSI (38.2\% vs $30.4 \%)$. Catheterrelated infection was more often diagnosed in PAC ( $41.5 \%$ vs $25.4 \%, p<0.001)$, although IE (2.8\% vs $10.9 \%, p<0.001)$ and osteoarticular infections $(2.3 \%$ vs $14.3 \%, p<0.001)$ were less frequent in these patients. PAC were less likely to have indwelling foreign material in place, such as osteoarticular prosthesis $(1.8 \%$ vs $10.5 \%, p<0.001)$ and cardiac devices $(0.5 \%$ vs $7.4 \%, p<0.001)$. PAC presented more thrombocytopenia, and less sepsis, persistent bacteremia, and metastatic infections. Echocardiography was performed more often in PWC $(43.7 \%$ vs $75.8 \%, p<0.001)$.

\section{Risk Factors for Infective Endocarditis}

Eighty-nine patients $(9.1 \%)$ were diagnosed with IE. Among them, six (6.7\%) were PAC, four with hematological malignancy and two with solid cancer. Clinical characteristics of PAC with IE are detailed in Table 1 of the supplementary material.

Compared with patients with other sources of BSI in the univariate analysis, patients with IE had more previous cardiopathy $(49.4 \%$ vs $16.5 \%, p<0.001)$, valvulopathy $(37.5 \%$ vs $14.5 \%, p<0.001)$, cardiac devices $(21.3 \%$ vs $4.3 \%, p<0.001)$, and presented more sepsis at presentation $(44.9 \%$ vs $31.2 \%, p=0.008)$, whereas they had less often an underlying malignant disease $(6.7 \%$ vs $23.7 \%, p<0.001)$. In the multivariate analysis, the presence of previous cardiopathy (aOR 4.392; 95\% CI 2.719-7.094, $\quad p<0.001)$ and persistent

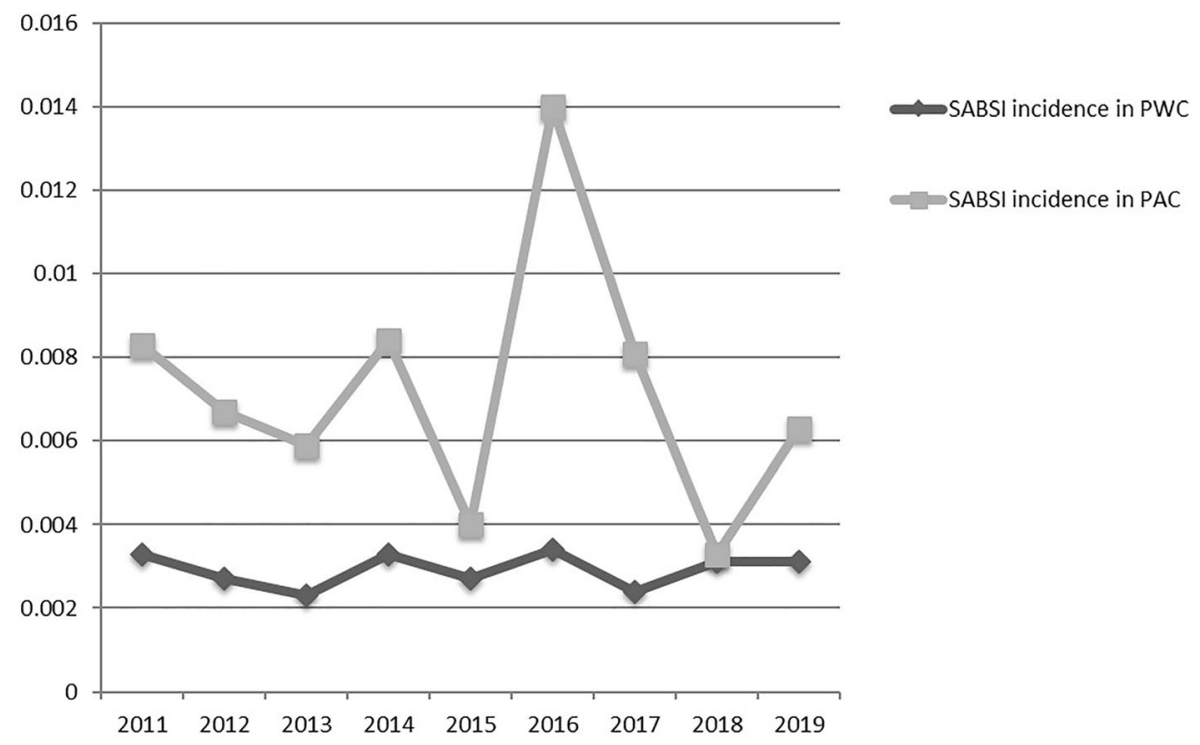

Fig. 1 Incidence of S. aureus bloodstream infection. SABSI Staphylococcus aureus bloodstream infection, PWC patients without cancer, PAC patients with active cancer 
Table 1 Baseline characteristics of patients with $S$. aureus bloodstream infection

\begin{tabular}{|c|c|c|c|c|}
\hline Variables & Total $(n=978)$ & No malignancy $(n=761)$ & Malignancy $(n=217)$ & $p$ values \\
\hline Age, years, mean (SD) & $64.6(15.9)$ & $66.1(15.8)$ & $59.5(15.2)$ & 0.001 \\
\hline Age $>65$ years & $521(53.3)$ & $441(58.0)$ & $80(36.9)$ & $<0.001$ \\
\hline Male sex & $647(66.3)$ & $504(66.4)$ & $143(65.9)$ & 0.890 \\
\hline \multicolumn{5}{|l|}{ Comorbidities } \\
\hline \multicolumn{5}{|l|}{ Charlson comorbidity index } \\
\hline Mean (SD) & $5.4(2.89)$ & $5.3(2.8)$ & $5.9(3.2)$ & 0.007 \\
\hline Charlson $>6$ points & $338(34.6)$ & $249(32.7)$ & $89(41.0)$ & 0.023 \\
\hline Cardiopathy & $191(19.5)$ & $171(22.5)$ & $20(9.2)$ & $<0.001$ \\
\hline Valvulopathy & $155(16.7)$ & $136(18.9)$ & $19(9.1)$ & 0.001 \\
\hline Diabetes mellitus & $327(33.4)$ & $296(38.9)$ & $31(14.3)$ & $<0.001$ \\
\hline COPD & $190(19.9)$ & $164(22.2)$ & $26(12.0)$ & 0.001 \\
\hline Chronic renal failure & $217(22.7)$ & $202(27.4)$ & $15(6.9)$ & $<0.001$ \\
\hline Neurological disorders & $122(12.8)$ & $115(15.6)$ & $7(3.2)$ & $<0.001$ \\
\hline Previous corticosteroids & $177(19.1)$ & $97(13.5)$ & $80(37.9)$ & $<0.001$ \\
\hline Antiplatelet drugs & $238(24.7)$ & $220(29.3)$ & $18(8.5)$ & $<0.001$ \\
\hline Anticoagulant drugs & $141(16.4)$ & $128(17.1)$ & $13(11.9)$ & 0.176 \\
\hline \multicolumn{5}{|l|}{ Acquisition } \\
\hline Community acquired & $229(23.4)$ & $213(28.0)$ & $16(7.4)$ & $<0.001$ \\
\hline Healthcare related & $435(44.5)$ & $317(41.7)$ & $118(54.4)$ & $<0.001$ \\
\hline Nosocomial & $314(32.1)$ & $231(30.3)$ & $83(38.2)$ & $<0.001$ \\
\hline MRSA & $207(21.2)$ & $170(22.3)$ & $37(17.1)$ & 0.093 \\
\hline \multicolumn{5}{|l|}{ Source of bloodstream infection } \\
\hline Catheter-related infection & $283(28.9)$ & $193(25.4)$ & $90(41.5)$ & $<0.001$ \\
\hline Infective endocarditis & $89(9.1)$ & $83(10.9)$ & $6(2.8)$ & $<0.001$ \\
\hline Ostheoarticular infection & $114(11.7)$ & $109(14.3)$ & $5(2.3)$ & $<0.001$ \\
\hline SSTI & $148(15.1)$ & $109(14.3)$ & $39(18.0)$ & 0.186 \\
\hline Urinary tract infection & $55(5.6)$ & $45(5.9)$ & $10(4.6)$ & 0.462 \\
\hline Unknown & $136(13.9)$ & $100(13.1)$ & $36(16.6)$ & 0.195 \\
\hline \multicolumn{5}{|l|}{ Indwelling foreign materials } \\
\hline Ostheoarticular prosthesis & $84(8.6)$ & $80(10.5)$ & $4(1.8)$ & $<0.001$ \\
\hline Cardiac device & $57(5.8)$ & $56(7.4)$ & $1(0.5)$ & $<0.001$ \\
\hline Thrombocytopenia $<100,000 / \mu \mathrm{L}$ & $179(18.3)$ & $78(10.3)$ & $101(46.1)$ & $<0.001$ \\
\hline Thrombocytopenia $<50,000 / \mu \mathrm{L}$ & $85(8.7)$ & $21(2.8)$ & $64(29.5)$ & $<0.001$ \\
\hline
\end{tabular}


Table 1 continued

\begin{tabular}{|c|c|c|c|c|}
\hline Variables & Total $(n=978)$ & No malignancy $(n=761)$ & Malignancy $(n=217)$ & $p$ values \\
\hline Thrombocytopenia $<20,000 / \mu \mathrm{L}$ & $34(3.5)$ & $2(0.3)$ & $32(14.7)$ & $<0.001$ \\
\hline \multicolumn{5}{|l|}{ Severity illness } \\
\hline Sepsis or septic shock & $317(32.4)$ & $276(36.3)$ & $41(18.9)$ & $<0.001$ \\
\hline Metastatic infection & $127(13.0)$ & $119(15.6)$ & $8(3.7)$ & $<0.001$ \\
\hline \multicolumn{5}{|l|}{ Infection management } \\
\hline Echocardiography & $484(67.2)$ & $405(75.8)$ & $79(42.5)$ & $<0.001$ \\
\hline Source control $(<72 \mathrm{~h})$ & $423 / 651(65.0)$ & $382 / 590(64.7)$ & $41 / 61(67.2)$ & 0.701 \\
\hline \multicolumn{5}{|l|}{ Outcomes } \\
\hline Persistent bacteremia & $172 / 856(20.1)$ & $143 / 663(21.6)$ & $29 / 193(15.0)$ & 0.026 \\
\hline 30-day mortality & $242 / 978(24.7)$ & $189 / 761(25.5)$ & $53 / 217(24.2)$ & 0.282 \\
\hline
\end{tabular}

Data are presented as no. (\%), unless otherwise specified

Statistically significant differences are marked in bold

$S D$ standard deviation, $C O P D$ chronic obstructive pulmonary disease, MRSA methicillin-resistant S. aureus, SSTI skin and soft tissues infection

bacteremia (aOR 3.545, 95\% CI 2.159-5.820, $p<0.001)$ were the only risk factors independently associated with IE, whereas active cancer was identified as a protective factor (aOR 0.338, 95\% CI 0.142-0.806, $p=0.011$ ) (Table 2). Goodness of fit, assessed by the Hosmer-Lemeshow test, was 0.277 . This protective effect was also observed when solid organ cancer and hematological malignancies were analyzed separately (data not shown). As shown in Table 2 of the supplementary material, no significant differences were observed between PWC and PAC with IE.

\section{Outcomes}

All-cause 30-day mortality among 978 episodes of SABSI was $24.6 \%$. No differences were found between PWC and PAC. After multivariate analysis, risk factors that remained independently associated with 30-day mortality were age $>65$ years, Charlson $>6$ points (aOR 1.881; 95\% CI 1.265-2.797; $p=0.002)$, unknown source of infection (aOR 2.759; 95\% CI 1.650-4.611, $p<0.001$ ), sepsis (aOR 7.578; 95\% CI 5.177-11.090, $p<0.001$ ), infection due to methicillin-resistant $S$. aureus (MRSA) strain (aOR 1.619; 95\% CI 1.006-2.608, $p=0.047$ ), and persistent bacteremia (aOR 5.666; 95\% CI $3.314-9.687 ; p<0.001$ ) (Table 3 in the supplementary material).

Among PAC, patients who died within 30 days were older, more often had solid organ cancer, unknown source of BSI, MRSA infection, sepsis and persistent bacteremia, while they had less frequently catheter-related infection and prompt source control. In the multivariate analysis, risk factors independently associated with 30-day mortality were solid organ cancer (aOR 3.239, 95\% CI 1.557-6.739, $p=0.002$ ), unknown source of infection (aOR 5.566; 95\% CI 2.146-14.443, $p<0.001$ ), and MRSA infection (aOR 4.240, 95\% CI 1.808-9.943, $p=0.001$ ) (Table 4 in the supplementary material). No PAC had SABSI relapse, while it occurred in seven PWC.

\section{DISCUSSION}

The present study, the first cohort study to compare SABSI and IE in patients with cancer and the general population, found IE to be 
Table 2 Univariate and multivariate analyses for risk factors for endocarditis

\begin{tabular}{|c|c|c|c|c|c|c|}
\hline Variable & $\begin{array}{l}\text { Patients without endocarditis } \\
(n=889)\end{array}$ & $\begin{array}{l}\text { Patients with endocarditis } \\
(n=89)\end{array}$ & $p$ value & OR & $95 \% \mathrm{CI}$ & $p$ value \\
\hline Age $>65$ years & $471(53.0)$ & $50(56.2)$ & 0.564 & & & \\
\hline Cardiopathy & $147(16.5)$ & $44(49.4)$ & $<0.001$ & 4.392 & $2.719-7.094$ & $<0.001$ \\
\hline Cardiac devices & $38(4.3)$ & $19(21.3)$ & $<0.001$ & & & \\
\hline Valvulopathy & $122(14.5)$ & $33(37.5)$ & $<0.001$ & & & \\
\hline Malignancy & $211(23.7)$ & $6(6.7)$ & $<0.001$ & 0.338 & $0.142-0.806$ & 0.014 \\
\hline Charlson $>6$ & $311(35.0)$ & $27(30.3)$ & 0.38 & & & \\
\hline $\begin{array}{l}\text { Antiplatelet } \\
\text { drug }\end{array}$ & $212(24.3)$ & $26(29.2)$ & 0.305 & & & \\
\hline $\begin{array}{l}\text { Platelet } \\
\qquad<50,000 / \mu \mathrm{L}\end{array}$ & $80(9.0)$ & $5(5.6)$ & 0.191 & & & \\
\hline $\begin{array}{l}\text { Platelet } \\
\qquad<20,000 / \mu \mathrm{L}\end{array}$ & $33(3.7 \%)$ & $1(1.1)$ & 0.167 & & & \\
\hline Sepsis & $277(31.2)$ & $40(44.9)$ & 0.008 & 1.371 & $0.838-2.242$ & 0.209 \\
\hline MRSA & $196(22.0)$ & $11(12.4)$ & 0.033 & & & \\
\hline $\begin{array}{l}\text { Persistent } \\
\text { bacteremia }\end{array}$ & $130(14.8)$ & $41(46.1)$ & $<0.001$ & 3.545 & $2.159-5.820$ & $<0.001$ \\
\hline
\end{tabular}

Data are presented as no. (\%), unless otherwise specified

Statistically significant differences are marked in bold

$O R$ odds ratio, $C I$ confidence interval, MRSA methicillin-resistant S. aureus

significantly less frequent in PAC, probably because of notable differences in the source of SABSI, baseline comorbidities, and site of acquisition between the groups.

In agreement with previous work, we found a higher incidence of SABSI in PAC than in PWC [24]. PAC are highly susceptible to SABSI as a result of their frequent hospital admissions, chemotherapy administration, common use of long-term indwelling catheters, and other invasive procedures [25]. In fact, in our cohort, PAC had more catheter-related BSI and a higher rate of healthcare-related or nosocomial SABSI acquisition, showing a clear relationship of this population with healthcare exposure.

The incidence of IE in patients with cancer and SABSI ranges between $1.6 \%$ and $6 \%$ $[15,16]$. Despite the higher incidence of SABSI in PAC, in our cohort few patients $(6 / 217,2.7 \%)$ in this group developed infective IE. A number of reasons may explain this finding. First of all, PAC with SABSI presented different epidemiological and clinical characteristics with respect to PWC: PAC were younger, had less comorbidity (including cardiopathy and cardiac or orthopedic devices), and had more catheter-related, healthcare-associated, and nosocomial SABSI. Indeed, PAC with SABSI exhibited a faster clearance of bacteremia than PWC. All these features have previously been found to be associated with a lower risk of IE [26, 27]. Second, an appealing pathogenic explanation of the lower frequency of IE could be the higher prevalence of thrombocytopenia in PAC. Platelet aggregation has a fundamental role in the formation of the vegetation. It has recently 
been demonstrated that activation of endothelial cells stimulates the release of von Willebrand factor multimers which bind circulating platelets as well as S. aureus [28]. Moreover, the presence of platelets increases the adhesion of $S$. aureus to collagen under flow conditions, overcoming shear stress [28, 29]. Of note, thrombocytopenia was present in half of the PAC in our cohort using a cutoff of $100,000 / \mu \mathrm{L}$, and it was more frequent in this group even when more restrictive cutoff values were used $(50,000 / \mu \mathrm{L}$ or $20,000 / \mu \mathrm{L})$. Although thrombocytopenia may have a protective role in PAC, we did not find this factor to be independently associated to the risk of developing IE. Moreover, previous studies in experimental models of IE have demonstrated that antiplatelet treatment may also hinder vegetation formation [30]. Recently, the use of dabigatran was also associated with a lower risk of SABSI in a large cohort of patients and with a lower risk of IE in an animal model [30, 31]. Nevertheless, we were not able to demonstrate the use of either antiplatelet agents or anticoagulant as a protective factor for IE.

Regarding the diagnostic approach to SABSI in PAC, and as a result of the low incidence of IE in this group, the question of whether these patients should undergo systematic transesophageal echocardiography (TEE) is controversial. In a recent study of the role of echocardiography in patients with cancer and SABSI, the authors did not find any additional infective IE after performing subsequent TEE [16]. Our study suggests that TEE is not mandatory in all PAC with SABSI and individualized indication should be carried out.

In our cohort, the 30-day mortality rate in PAC with SABSI was $24.2 \%$, a figure higher than reported in previous studies, typically between $16.7 \%$ and $21.2 \% \quad[12,15]$. Importantly, although PAC presented more low-risk SABSI, 30-day mortality in this group was as high as in PWC. Unlike a previous study that reported hematological malignancies as a risk factor for mortality in SABSI [15], we found that patients with solid organ cancer and unknown source of infection had a higher risk for 30-day mortality. Various explanations might be proposed for the higher mortality rate observed in patients with solid organ cancer. First, our cohort also included patients admitted to palliative care units, mostly with solid tumors. Second, the higher mortality rate might reflect more severe disease, given that the hospitals in our study are tertiary referral centers in Catalonia. Third, patients with hematological malignancy were younger and had less comorbidity than patients with solid organ cancer, features inversely associated with mortality.

This study has some strengths and limitations. It is, to the best of our knowledge, the first to compare SABSI and IE in PAC with those in patients without malignancy, and also the first to report the clinical characteristics and risk factors for S.aureus IE in this population. Although the study was conducted at two different centers, the large cohort of patients included increases the external validity of its results. The main limitation of this study was its retrospective design, although the patients were prospectively followed by infectious disease specialists. The low prevalence of IE in patients with cancer also hampered the multivariate analysis of risk factor for IE in this specific group. Because PAC were closely monitored, SABSI could be detected, diagnosed, and treated earlier in the course of the disease in PAC than in PWC and it could therefore be prone to selection bias.

\section{CONCLUSIONS}

PAC showed a higher incidence of SABSI and IE was detected less frequently than in PWC. Relevant differences in sources of SABSI, baseline comorbidities, and site of acquisition could explain the differential risk observed between populations. Thirty-day mortality was high in both groups of patients, underlying the importance of prompt source control and optimal antibiotic treatment. Our study suggests that PAC without previous cardiopathy and with rapid clearance of bacteremia have a low risk of developing IE. Thus, it is necessary to individualize the evaluation of the necessity of TEE in selected patients. This may reduce costs, length of stay, and avoid possible complications. 


\section{ACKNOWLEDGEMENTS}

Funding. This work was supported by Plan Nacional de I+D+i 2017-2021 and Instituto de Salud Carlos III, Subdirección General de Redes y Centros de Investigación Cooperativa, Ministerio de Economía, Industria y Competitividad, Spanish Network for Research in Infectious Diseases (REIPI RD16/0016/0005; RD16/0016/ 0002; RD16/0016/0001) and was co-financed by the European Development Regional Fund 'A way to achieve Europe', Operative program Intelligent Growth 2014-2020 and by Centro de Investigación Biomédica en Red (CIBER) de Enfermedades Respiratorias (CB06/06/0037), and CIBER de Enfermedades Infecciosas (CB/21/ 13/00009), Instituto de Salud Carlos III, Madrid, Spain. The Rapid Service Fee was funded by the authors.

We thank the CERCA Programme / Generalitat de Catalunya for institutional support.

Editorial Assistance. We thank Mike Maudsley from the University of Barcelona for the English editing of this manuscript. His service was funded by the authors.

Authorship. All named authors meet the International Committee of Medical Journal Editors (ICMJE) criteria for authorship for this article, take responsibility for the integrity of the work as a whole, and have given their approval for this version to be published.

Authors' Contribution. SG, JL, MT and CG had full access to all of the data of the study and took responsibility for the integrity of the data and the accuracy of the data analysis. SG and CG conceived and designed the study, collected and analyzed the data, wrote and revised the manuscript. IG, MP, CG and JC designed the study and critically reviewed the manuscript.

Disclosures. Sara Grillo received a grant from the Spanish Network for Research in Infectious Diseases (REIPI RD16/0016/0005; RD16/0016/0002; RD16/0016/0001). Guillermo Cuervo, Julia Laporte-Amargòs, Manel Tuells, Immaculada Grau, Damaris Berbel, Carlota
Gudiol, Miquel Pujol and Jordi Carratalà have nothing to disclose.

Compliance with Ethics Guidelines. This study was approved by the Clinical Research Ethics Committee of Bellvitge University Hospital (PR326/21).

Data Availability. The datasets generated during and/or analyzed during the current study are available from the corresponding author on reasonable request.

Open Access. This article is licensed under a Creative Commons Attribution-NonCommercial 4.0 International License, which permits any non-commercial use, sharing, adaptation, distribution and reproduction in any medium or format, as long as you give appropriate credit to the original author(s) and the source, provide a link to the Creative Commons licence, and indicate if changes were made. The images or other third party material in this article are included in the article's Creative Commons licence, unless indicated otherwise in a credit line to the material. If material is not included in the article's Creative Commons licence and your intended use is not permitted by statutory regulation or exceeds the permitted use, you will need to obtain permission directly from the copyright holder. To view a copy of this licence, visit http://creativecommons.org/licenses/bync/4.0/.

\section{REFERENCES}

1. Laupland KB, Lyytikäinen O, Søgaard M, et al. The changing epidemiology of Staphylococcus aureus bloodstream infection: a multinational populationbased surveillance study. Clin Microbiol Infect. 2013. https://doi.org/10.1111/j.1469-0691.2012. 03903.x.

2. Souli M, Ruffin F, Choi SH, et al. Changing characteristics of Staphylococcus aureus Bacteremia: results from a 21-year, prospective, longitudinal study. Clin Infect Dis. 2019;69:1868-77. https:// doi.org/10.1093/cid/ciz112.

3. van Hal SJ, Jensen SO, Vaska VL, Espedido BA, Paterson DL, Gosbell IB. Predictors of mortality in 
Staphylococcus aureus bacteremia. Clin Microbiol Rev. 2012;25:362-86. https://doi.org/10.1128/ CMR.05022-11.

4. Habib G, Erba PA, Iung B, et al. Clinical presentation, aetiology and outcome of infective endocarditis. Results of the ESC-EORP EURO-ENDO (European Infective Endocarditis) registry: a prospective cohort study. Eur Heart J. 2019;40: 3222-3232B. https://doi.org/10.1093/eurheartj/ ehz620.

5. Østergaard L, Bruun NE, Voldstedlund M, et al. Prevalence of infective endocarditis in patients with positive blood cultures: a Danish nationwide study. Eur Heart J. 2019;40:3237-44. https://doi.org/10. 1093/eurheartj/ehz327.

6. Rieg S, Peyerl-Hoffmann G, de With K, et al. Mortality of $S$. aureus bacteremia and infectious diseases specialist consultation-a study of 521 patients in Germany. J Infect. 2009;59:232-9. https://doi.org/ 10.1016/j.jinf.2009.07.015.

7. van Hal SJ, Jones M, Gosbell IB, Paterson DL. Vancomycin heteroresistance is associated with reduced mortality in ST239 methicillin-resistant Staphylococcus aureus blood stream infections. PLoS ONE. 2011. https://doi.org/10.1371/journal.pone. 0021217 .

8. Sung H, Ferlay J, Siegel RL, et al. Global Cancer Statistics 2020: GLOBOCAN estimates of incidence and mortality worldwide for 36 cancers in 185 countries. CA Cancer J Clin. 2021;71:209-49. https://doi.org/10.3322/caac. 21660.

9. Martin GS, Mannino DM, Eaton S, Moss M. The epidemiology of sepsis in the United States from 1979 through 2000. N Engl J Med. 2003;348: 1546-54. https://doi.org/10.1056/nejmoa022139.

10. Primo M, Guilarde A, Martelli C, Batista L, Turchi M. Healthcare-associated Staphylococcus aureus bloodstream infection: length of stay, attributable mortality, and additional direct costs. Braz J Infect Dis. 2012;16:503-9. https://doi.org/10. 1016/J.BJID.2012.10.001.

11. Kang CI, Song JH, Chung DR, et al. Bloodstream infections in adult patients with cancer: clinical features and pathogenic significance of Staphylococcus aureus bacteremia. Support Care Cancer. 2012;20:2371-8. https://doi.org/10.1007/s00520011-1353-z.

12. Wisplinghoff H, Seifert H, Wenzel RP, Edmond MB. Current trends in the epidemiology of nosocomial bloodstream infections in patients with hematological malignancies and solid neoplasms in hospitals in the United States. Clin Infect Dis. 2003;36: 1103-10. https://doi.org/10.1086/374339.
13. Marín M, Gudiol C, Garcia-Vidal C, Ardanuy C, Carratalà J. Bloodstream infections in patients with solid tumors: epidemiology, antibiotic therapy, and outcomes in 528 episodes in a single cancer center. Medicine (US). 2014;93:143-9. https://doi.org/10. 1097/MD.0000000000000026.

14. González-Barca E, Carratalá J, Mykietiuk A, Fernández-Sevilla A, Gudiol F. Predisposing factors and outcome of Staphylococcus aureus bacteremia in neutropenic patients with cancer. Eur J Clin Microbiol Infect Dis. 2001;20:117-9. https://doi. org/10.1007/PL00011241.

15. Bello-Chavolla OY, Bahena-Lopez JP, GarciadiegoFosass $\mathrm{P}$, et al. Bloodstream infection caused by $S$. aureus in patients with cancer: a 10-year longitudinal single-center study. Support Care Cancer. 2018;26:4057-65. https://doi.org/10.1007/s00520018-4275-1.

16. Loh Z, Estacio O, Grigg A, Holmes NE, Chong G, Hawkes EA. Echocardiography has low utility in cancer patients with Staphylococcus aureus bacteraemia: findings from a retrospective study. Support Care Cancer. 2018;26:3083-9. https://doi.org/10. 1007/s00520-018-4162-9.

17. Grillo $S$, Cuervo $G$, Carratalà $J$, et al. Impact of $\beta$ lactam and daptomycin combination therapy on clinical outcomes in methicillin-susceptible Staphylococcus aureus bacteremia: a propensity scorematched analysis. Clin Infect Dis. 2019;69:1480-8. https://doi.org/10.1093/cid/ciz018.

18. Grillo S, Cuervo G, Carratalà J, et al. Characteristics and outcomes of staphylococcus aureus bloodstream infection originating from the urinary tract: a multicenter cohort study. Open Forum Infect Dis. 2020. https://doi.org/10.1093/ofid/ofaa216.

19. Charlson ME, Pompei P, Ales KL, MacKenzie CR. A new method of classifying prognostic comorbidity in longitudinal studies: development and validation. J Chronic Dis. 1987;40:373-83. https://doi. org/10.1016/0021-9681(87)90171-8.

20. Friedman ND, Kaye KS, Stout JE, et al. Health careassociated bloodstream infections in adults: a reason to change the accepted definition of community-acquired infections. Ann Intern Med. 2002. https://doi.org/10.1097/00019048-20020600000016.

21. Singer M, Deutschman CS, Seymour C, et al. The third international consensus definitions for sepsis and septic shock (Sepsis-3). JAMA. 2016;315: 801-10. https://doi.org/10.1001/jama.2016.0287.

22. Li JS, Sexton DJ, Mick N, et al. Proposed modifications to the Duke criteria for the diagnosis of 
infective endocarditis. Clin Infect Dis. 2000;30: 633-8. https://doi.org/10.1086/313753.

23. European Committee on Antimicrobial Susceptibility Testing Breakpoint tables for interpretation of MICs and zone diameters n.d. 2021, v 11.0. https:// www.eucast.org/clinical_breakpoints/.

24. Skov R, Gottschau A, Skinhøj P, et al. Staphylococcus aureus bacteremia: a 14-year nationwide study in hematological patients with malignant disease or agranulocytosis. J Infect Dis. 1995;27:563-8. https://doi.org/10.3109/00365549509047068.

25. Crawford J, Dale DC, Lyman GH. Chemotherapyinduced neutropenia: risks, consequences, and new directions for its management. Cancer. 2004;100: 228-37. https://doi.org/10.1002/cncr.11882.

26. Palraj BR, Baddour LM, Hess EP, et al. Predicting risk of endocarditis using a clinical tool (PREDICT): scoring system to guide use of echocardiography in the management of Staphylococcus aureus bacteremia. Clin Infect Dis. 2015;61:18-28. https://doi. org/10.1093/cid/civ235.

27. Hill EE, Vanderschueren S, Verhaegen J, et al. Risk factors for infective endocarditis and outcome of patients with Staphylococcus aureus bacteremia. Mayo Clin Proc. 2007;82:1165-9. https://doi.org/ 10.4065/82.10.1165.
28. Liesenborghs L, Meyers S, Lox M, et al. Staphylococcus aureus endocarditis: distinct mechanisms of bacterial adhesion to damaged and inflamed heart valves. Eur Heart J. 2019;40:3248-59. https://doi. org/10.1093/eurheartj/ehz175.

29. Claes J, Peetermans M, Liesenborghs L, et al. Adhesion of Staphylococcus aureus to the vessel wall under flow is mediated by von Willebrand factorbinding protein. Blood. 2014;124:1669-76. https:// doi.org/10.1182/blood-2014-02-558890.

30. Veloso TR, Que Y-AA, Chaouch A, et al. Prophylaxis of experimental endocarditis with antiplatelet and antithrombin agents: a role for long-term prevention of infective endocarditis in humans? J Infect Dis. 2015;211:72-9. https://doi.org/10.1093/infdis/ jiu426.

31. Butt JH, Fosbøl EL, Verhamme P, et al. Dabigatran and the risk of Staphylococcus aureus bacteremia: a nationwide cohort study. Clin Infect Dis. 2021;73: 480-6. https://doi.org/10.1093/cid/ciaa661.

\section{Publisher's Note}

Springer Nature remains neutral with regard to jurisdictional claims in published maps and institutional affiliations 